\title{
Analysis of defect capture cross sections using non-radiative multiphonon-assisted trapping model
}

\author{
Davide Garetto a,b,*, Yoann Mamy Randriamihaja ${ }^{c}$, Alban Zaka ${ }^{c}$, Denis Rideau ${ }^{c}$, Alexandre Schmid ${ }^{\mathrm{b}}$, \\ Hervé Jaouen ${ }^{\mathrm{c}}$, Yusuf Leblebici ${ }^{\mathrm{b}}$ \\ ${ }^{a}$ IBM Systems and Technology Group - 850, rue Jean Monnet, Crolles, France \\ ${ }^{\mathrm{b}}$ Ecole Polytechnique Fédérale de Lausanne - Station 11, Lausanne, Switzerland \\ ${ }^{\mathrm{c}}$ STMicroelectronics - 850, rue Jean Monnet, Crolles, France
}

\section{A R T I C L E I N F O}

Article history:

Available online 25 November 2011

\section{Keywords:}

Trap cross-section

Multiphonon trapping model

AC analysis

\begin{abstract}
A B S T R A C T
A multiphonon-assisted model included in a Poisson-Schroedinger solver has been applied to the calculation of the capture/emission trapping rates of CMOS oxide interface defects. The dependencies of trap capture cross-sections with trap energy, depth, applied bias and temperature have been extracted, with the purpose of evaluating the accuracy of constant cross-section models adopted in compact and empirical approaches. The model has been applied to the extraction of interface trap concentrations and to the accurate $\mathrm{AC}$ analysis of the trap frequency response.
\end{abstract}

(c) 2011 Elsevier Ltd. All rights reserved.

\section{Introduction}

CMOS device performances are considerably affected by the degradation of the oxide layers and $\mathrm{Si} / \mathrm{SiO}_{2}$ interfaces, causing uncontrolled parameter variations and general device lifetime reduction $[1,2]$. Physical models describing the oxide degradation mechanisms and defects effects on performances are growing in importance in modern nanoscale technologies [3-5].

A common example of interfacial point defects is represented by a $s p^{3}$-like silicon dangling bond formed at the $\mathrm{Si} / \mathrm{SiO}_{2}$ interface [6]; it has been historically characterized using Electron Paramagnetic Resonance (EPR) spectroscopy $[7,8]$. The amphoteric nature of $P_{b}$ centers is responsible of the altered electrical characteristics as the defects act both as electron donor and acceptor impurities $[9,10]$. Nevertheless, the relation between microscopic physical aspects and macroscopic effects on the electrical characteristics is still under debate [11]. Recently, correlation has been found between Random Telegraph Noise (RTN) and Negative Bias Temperature Instability (NBTI), by considering interface and oxide defects having a wide spread distribution of capture/emission (C/E) rates [12]. It has also been shown that the trapping dynamics of single defects are playing a major role in the phenomena [11], while $C / E$ transitions have been attributed to multiphononassisted mechanisms [13]. For these reasons, increasing effort has

\footnotetext{
* Corresponding author at: IBM Systems and Technology Group - 850, rue Jean Monnet, Crolles, France.

E-mail address: david.garetto@fr.ibm.com (D. Garetto).
}

been applied to the determination of the relationship between the macroscopic time constants and the microscopic variables predicted by multiphonon theory.

Compact approaches rely on effective trap cross-sections to model the C/E tunneling probabilities, but their dependence on temperature, trap energy and electrical field are often neglected or calculated with empirical models [14]. Multiphonon-assisted inelastic transitions are considered an important ingredient when dealing with tunneling interaction events between the carrier reservoirs, e.g. the channel of a MOSFET, and the point defect position. Quantum models have been proposed for the analysis of trap-assisted tunneling (TAT) between the channel states and a neutral trap level $[3,15]$.

In this work a multiphonon-assisted trapping model integrated into a Poisson-Schroedinger (PS) solver has been applied to the investigation of the dynamics of charge trapping and to the extraction of the C/E time constants for both carriers in different operating regimes. Section 2 covers the model description; the $\mathrm{C} / \mathrm{E}$ frequencies and the effective trap cross-sections are detailed in Section 3; finally the calculation of the intrinsic trap frequency and its impact on MOS impedance are discussed in Section 4.

\section{Model description and validation}

A 1D multiphonon-assisted trapping model included in a selfconsistent Poisson-Schroedinger k.p solver [16] has been adopted. The quasi-Fermi level $E_{F}$ of a trap placed at position $x$ and energy $E_{T}$ in the oxide layer needs to be calculated in order to determine the trapped charge $\rho_{T}$. The non-linear rate equation is solved: 


$$
\begin{aligned}
\frac{\partial \rho_{T}\left(x, E_{T}, E_{F}, t\right)}{\partial t}= & \Phi_{c}\left(x, E_{T}, E_{F}, t\right)-\Phi_{e}\left(x, E_{T}, E_{F}, t\right) \\
& +\widetilde{\Phi}_{e}\left(x, E_{T}, E_{F}, t\right)-\widetilde{\Phi}_{c}\left(x, E_{T}, E_{F}, t\right),
\end{aligned}
$$

where $\Phi_{c} / \Phi_{e}\left(\widetilde{\Phi}_{c} / \widetilde{\Phi}_{e}\right)$ are the C/E fluxes from the reservoir to the trap position for $e^{-}\left(h^{+}\right.$, respectively). The equation has been solved in steady-state conditions $\left(\partial \rho_{T} / \partial t=0\right)$ for DC analysis, and in the Fourier domain for AC analysis following the small-signal model in [17].

The dynamics of multiphonon trapping and tunneling mechanisms are included in the $e^{-} \mathrm{C} / \mathrm{E}$ frequencies $\tau_{C}^{-1} / \tau_{E}^{-1}$. These characteristic frequencies used for the calculation of the fluxes $\Phi$ can be determined with:

$\tau_{C}^{-1}\left(x, E_{T}\right)=\int_{\mathscr{E}} W_{c}\left(x, \mathscr{E}, E_{T}\right) f(x, \mathscr{E}) d \mathscr{E}$,

$\tau_{E}^{-1}\left(x, E_{T}\right)=\tau_{C}^{-1}\left(x, E_{T}\right) e^{\frac{E_{T}-\mathcal{E}}{k_{B} T}}$

where $f$ is the channel carrier distribution, $\mathscr{E}$ is the energy of the carrier in the substrate and $T$ is the temperature. Deriving the capture probabilities from multiphonon theory $[14,15,18]$ one has:

$$
\begin{aligned}
W_{c}\left(x, \mathscr{E}, E_{T}\right)= & \frac{2 \pi}{h} R(\Delta E)|V|^{2} \\
& \times \exp \left(\frac{F^{2}}{F_{C}^{2}}\right)\left[r S\left(1-\frac{\Delta E}{h \omega S}\right)^{2}+(1-r) \sqrt{\left(\frac{\Delta E}{h \omega S}\right)^{2}+4 \bar{n}(\bar{n}+1)}\right] .
\end{aligned}
$$

in which $F$ is the electrical field in the structure, $\Delta E=\mathscr{E}-E_{T}$ and $\bar{n}$ is given by the Bose-Einstein distribution of phonons. The term $R(\Delta E)$ is expressed as:

$R(\Delta E)=\frac{1}{h \omega} \exp \left[-(2 \bar{n}+1) S+\frac{\Delta E}{2 k_{B} T}\right] \sum_{m} I_{m}(z) \delta(m h \omega-\Delta E)$,

being $I_{m}(z)$ the reduced Bessel function of order $m$ and $\delta(x)$ the Dirac delta function. The factor $r$ within the multiphonon theory can be calculated from $r=\left|V_{\Lambda}\right|^{2} /\left(|V|^{2} S\right)$, with $|V|^{2}=\left.\left|<\Phi_{B}\right| W_{S}^{O}\left|\Phi_{A}\right\rangle\right|^{2}$ representing the wave-function overlap from the free state $\Phi_{A}$ to the bound state $\Phi_{B}$ through the perturbation $W_{s}^{O}$ calculated from the overlapping of the defect and trap wavefunctions as in [15], and $\left|V_{A}\right|^{2}=\left|<\Phi_{B}\right| W_{S}^{O}\left|\Phi_{A}>\right|\left(\left|<\Phi_{B}\right| W_{S}^{O}\left|\Phi_{B}>\right|-\left|<\Phi_{A}\right| W_{S}^{O} \mid \Phi_{A}>1\right)^{2}$

[19]. Cancellation of the first term in Eq. (3) appears when $\Delta E=\hbar \omega$ $S$. A constant value of 0.9 has been used in the calculation of all the cross-section simulations and AC results, to avoid discontinuities in the capture emission rates when $r=1.0$ and $\Delta E=\hbar \omega S .{ }^{1}$

A critical electric field factor $F_{C}$ has been introduced to reproduce the bias-dependence of trapping rates of bulk defects in the oxide layer in the high-voltage regime. The use of this empirical parameter is needed to reproduce single defects experiment results where the capture rates are evaluated from variation of electrical parameters and indicate a smaller dependence than exponential with the field [23]. Additional studies based on time dependent defect spectroscopy (TDDS) in [24] show how this behavior can reach saturation at higher stress voltages. Huard [23] showed that a constant critical field of $3 \mathrm{MV} / \mathrm{cm}$ agrees well with the capture rates exponential dependence measured over

\footnotetext{
1 This is related to the violation of the first order perturbation theory when, in a configuration-coordinate diagram, the lower parabola (bound state) crosses the upper one (free state) at its minimum, leading to a completely anharmonic lattice potential around this crossing point $[20,21]$. For practical implementation in a device simulator several authors $[22,3]$ replace this term by unity. The factor has been theoretically calculated in bulk materials adopting a Billiard-Ball model for the wavefunction, and, depending on the trap energy, it has been found varying from 0.5 to 1.0. Margina dependence on $r$ has been found on both the trapping rates and AC characteristics.
}

different CMOS technologies. The Huang-Rhys factor $S$ and the phonon frequency $\omega$ have been taken consistent to the values measured in [25] on the entire oxide layer ( $S=9.6$ and $h \omega=39.2 \mathrm{meV}$ ). A billiard-ball model $[14,15]$ has been adopted for calculating the trap wavefunction, while the energetic levels and the carrier wavefunctions are obtained from the solution of the Schroedinger equation. The wavefunction matching problem at the $\mathrm{SiO}_{2} / \mathrm{Si}_{x} \mathrm{O}_{2(1-x)} / \mathrm{Si}$ region has been treated with a finite difference approach within the envelope function approximation and using position dependent effective mass approximation model parameters [26]. Since the oxide and the interfacial region are amorphous materials whose exact nature is uncertain, this method represents an efficient and versatile way to capture the main features of the $\mathrm{SiO}_{2} /$ $\mathrm{Si}_{x} \mathrm{O}_{2(1-x)} / \mathrm{Si}$ interface region (finite band offsets, change of the effective masses, penetration of the waves in the oxide, ...), guaranteeing that the wavefunction and all its derivatives always remain continuous at the material interfaces [27]. The same considerations apply to the calculation of $\tilde{\tau}_{C} / \tilde{\tau}_{E}$ for $h^{+}$.

$\mathrm{Ab}$-initio and density functional theory (DFT) simulations indicate that the $\mathrm{Si} / \mathrm{SiO}_{2}$ interface should be considered as a gradual transition from bulk $\mathrm{Si}$ to bulk $\mathrm{SiO}_{2}$ more likely formed of a $\mathrm{Si}_{x} \mathrm{O}_{2(1-x)}$ material [28]. The structural and electrical properties of the band structure gradually vary between the two stochiometric bulk materials' properties. The extension of this region is known to be ranging from $2 \AA$ to $10 \AA[29,28]$ and to present a large number of unpassivated dangling bonds after electrical stress [30,31]. Consequently, a clear distinction between $P_{b}$ and $E^{\prime}$ defects is difficult to be established in such a region. In this study, the defects have been considered having an amphoteric nature to obtain a good correlation between the stretch-out DC effects in both accumulation and inversion, and the AC response in depletion. The considered microscopic trap parameters $(S, h \omega, \ldots)$ have been taken from measurement values based on scanning tunneling spectroscopy studies for single-defect Si dangling bonds [25]. The dynamics of other oxide traps $\left(E_{\gamma}\right.$ and $\left.E_{\delta}[7,32]\right)$ are better described including metastable states $[11,24]$. However, EPR studies indicated that these defects relax almost immediately after the removal of the stress condition [9], and thus they have not been investigated in this study. The considered trapping model is suitable for the modeling of amphoteric, acceptor and donor trap distributions. In particular, the amphoteric nature of $P_{b}$ centers (neutral when unoccupied and having the possibility to capture a $h^{+}$or an $e^{-}$) leads to:

$\rho_{T}\left(x, E_{T}\right)=N_{T}\left(x, E_{T}\right)\left(1-2 f_{T}\left(x, E_{T}\right)\right)$,

where $N_{T}\left(x, E_{T}\right)$ is the input defect distribution in the oxide layer and $f_{T}$ indicates the trap occupation given by the Fermi-Dirac distribution.

The model predictions have been compared to those obtained using a multiphonon capture model [15] applied to the study of TAT current. Same structure and model parameters as in [15] have been used for the comparison. A good qualitative alignment on multiphonon C/E frequencies calculated with Eq. (2) is observed (Fig. 1a). Quantitative discrepancies have been attributed to some differences between the model in [15] and this work. Indeed, we have considered both gate and substrate contributions in the rate equation, the impact of the electric field on $h^{+}$and $e^{-}$fluxes, inelastic emission towards both the gate and channel reservoirs and selfconsistent simulation of device electrostatics. The importance of computing a self-consistent device electrostatics is shown in the band diagram of Fig. 1b. The trapped charge modifies the potential profile in the structure and thus needs to be taken into account in the Poisson equation. For an accurate approach in all bias conditions, both gate and substrate fluxes should be taken into account considering also the $h^{+}$trapping currents towards the valence 

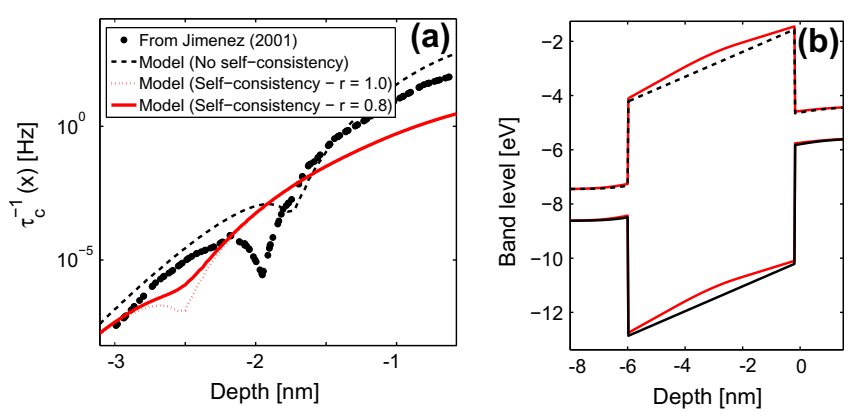

Fig. 1. (a) $e^{-}$Capture frequencies $\tau_{c}^{-1}$ vs. the position $x$ in the oxide calculated with the proposed model and with the model in [15]. Structure parameters are detailed in [15]. (b) Band diagram of the structure showing the conduction and valence bands bending induced by the negatively filled charged traps in the oxide layer.

bands. Inelastic and direct tunneling towards the gate can also occur with important effects on the TAT current and trap occupation.

\section{Capture/emission frequencies and cross-sections}

In this section, the $\mathrm{C} / \mathrm{E}$ frequencies and cross-sections are determined in a NMOS structure having $50 \AA$ oxide thickness. For all the simulations performed in this work, traps have been assumed in equilibrium conditions with the reservoirs at the DC bias conditions.

A theoretical study on the C/E rates dependence in energy, position and bias has been conducted. Fig. 2 shows a zoom of the oxide region in Fig. $1 \mathrm{~b}$ in weak inversion conditions $(V=0.9 \mathrm{~V})$. In (a), the higher probability of $e^{-}$of being captured in the vicinity of the conduction band is shown, where the tunneling transition is favorable due to the overlapping of carrier and trap wavefunctions. The energy dependence is explained considering that transition of carriers is less favorable when a larger amount of phonons is emitted/ absorbed. The emission rates in (b) show a stronger dependence in energy given by the detailed balance in Eq. (2). For the considered voltages, the oxide field remains smaller or comparable to the critical field $F_{C}$ and thus, this empirical parameter is assumed to have a reduced effect on the characteristics. A similar exponential decrease in energy and position has been observed for holes $\mathrm{C} /$ E rates.

The energy and depth dependencies of $\tau_{C}^{-1} / \tau_{E}^{-1}$ have been illustrated at the $\mathrm{Si} / \mathrm{SiO}_{2}$ interface ( $x=0$ in Fig. 2 ) and at the $\mathrm{Si}$ conduction/valence band edges $E_{\mathrm{CB}}^{\mathrm{Si}} / E_{\mathrm{VB}}^{\mathrm{Si}}$ in Fig. 3. The exponential dependence due to the wavefunction penetration in the oxide is

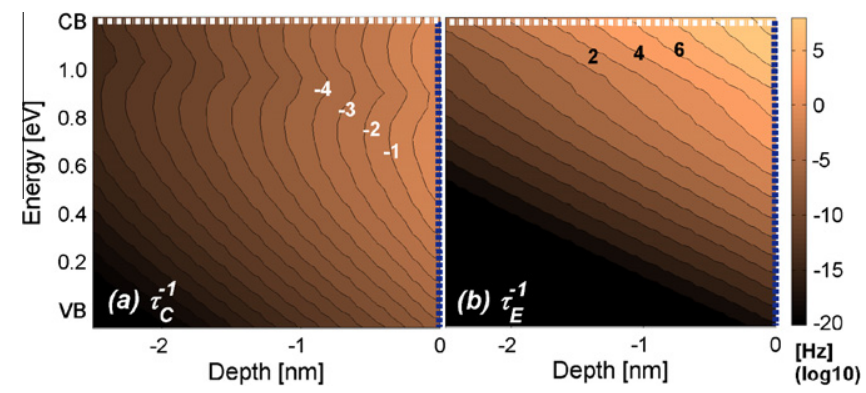

Fig. 2. Simulated capture (a) and emission (b) frequencies of electrons in weak inversion conditions $(V=0.9 \mathrm{~V})$ as a function of the trap energy and depth in the oxide layer. The oxide/substrate interface is placed at position $x=0$ with the substrate conduction and valence band edges at $0 \mathrm{eV}$ and $1.2 \mathrm{eV}$. A zoom of the band diagram in Fig. $1 \mathrm{~b}$ in proximity of the $\mathrm{Si} / \mathrm{SiO}_{2}$ interface is shown. Cuts in energy and depth are shown in Fig. 3. The predictions of the multiphonon-assisted model show a strong decrease of capture and emission rates in oxide depth and trap energy.
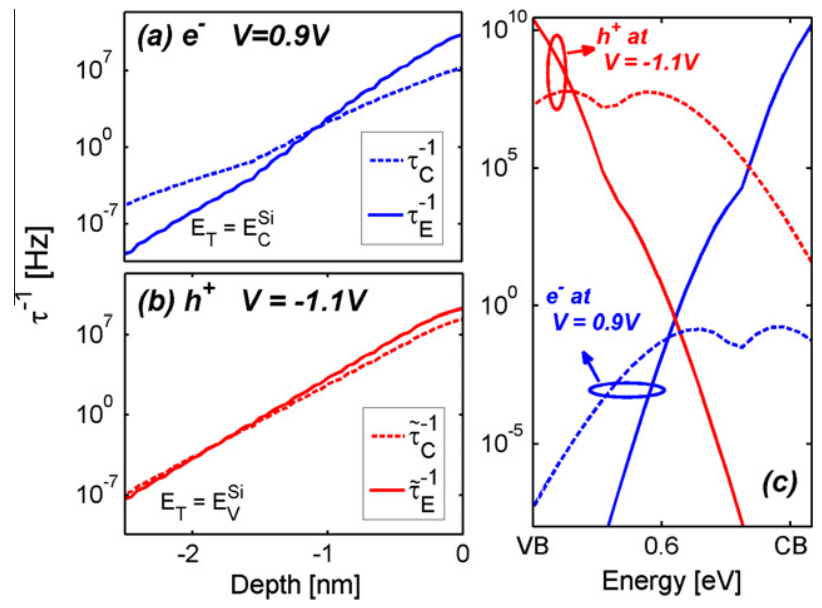

Fig. 3. Capture and emission rates as a function of the oxide depth ( $e^{-}$in (a) and $h^{+}$ in (b)) and the trap energy (c) related to the cuts of Fig. 2 along the dotted lines. The capture/emission frequencies are higher near the $\mathrm{Si} / \mathrm{SiO}_{2}$ interface. The hole capture/emission rates $\tilde{\tau}_{c} / \tilde{\tau}_{e}$ are higher in proximity of the $\mathrm{Si}$ valence band $E_{T}=E_{\mathrm{VB}}^{\mathrm{Si}}$, while $\tau_{c} / \tau_{e}$ peak near the conduction band $E_{T}=E_{\mathrm{CB}}^{\mathrm{Si}}=1.2 \mathrm{~V}$. The quantities referred to $h^{+}$are illustrated for $V=-1.1 \mathrm{~V}$, while those related to $e^{-}$for weak inversion conditions at $V=0.9 \mathrm{~V}$.

stronger than the dependence in energy. The $e^{-}$capture rate in weak inversion is of several orders of magnitude smaller than both the emission rate and the capture rate of holes in accumulation, due to the reduced inversion charge in the channel.

The dynamics of the system can also be described by the capture cross-section of the trap. Fig. 4 shows the effective capture cross-sections simulated for different bias conditions: electrons in weak (a) and strong inversion (b), and holes in accumulation (c), flat-band (d) and depletion (e) regimes. The classical definition of capture cross-section [33] has been adopted: $\sigma_{e}=\tau_{c}^{-1} /\left(v_{t h} n\right)$ and $\sigma_{h}=\tilde{\tau}_{C}^{-1} /\left(v_{t h} p\right)$, for $e^{-}$and $h^{+}$respectively, where $v_{t h}=10^{5} \mathrm{~m} /$ $\mathrm{s}$ is the carrier saturation velocity and $n$ and $p$ are the total mobile charge densities calculated by the Schroedinger solver. The $h^{+}$capture cross-section is considerably smaller than the one related to $e^{-}$, which increases from depletion to inversion.

The strong temperature dependence of the cross-sections has been illustrated in Fig. 5a. The calculated quantities for $h^{+}$(solid lines) and $e^{-}$(dashed lines) are shown as a function of oxide depth for three different temperatures $\left(-40^{\circ} \mathrm{C}, 25^{\circ} \mathrm{C}\right.$ and $\left.100^{\circ} \mathrm{C}\right)$, indicated in color. Fig. 5b also shows the energy dependence at the $\mathrm{Si} / \mathrm{SiO}_{2}$ interface. The two cross-sections are shown at an energy level $E_{T}=E_{V}^{\mathrm{Si}}$. The applied gate voltage in the structure is $V=0.9 \mathrm{~V}$ (weak inversion). In this regime, the temperature dependence of $\sigma_{h}$ is stronger than the one of $\sigma_{e}$. The investigated temperature dependence can also be modeled by a compact Arrhenius equation for the considered temperature range. However, such an approach would require extraction or calibration of empirical parameters.

\section{Applications and discussion}

\subsection{Trap frequency response}

The C/E rates of traps play a fundamental role in the frequency dependence of the MOS admittance. The intrinsic trap cut-off frequency $\tau_{0}^{-1}$, calculated as the sum of all $C / E$ frequencies [16], has been considered as the main indicator of the intrinsic trap response and its frequency dependence. Indeed, it represents the frequency limit at which traps are able to exchange carriers with the reservoir. Each iso-level in the contour plots of Fig. 6 represents the 

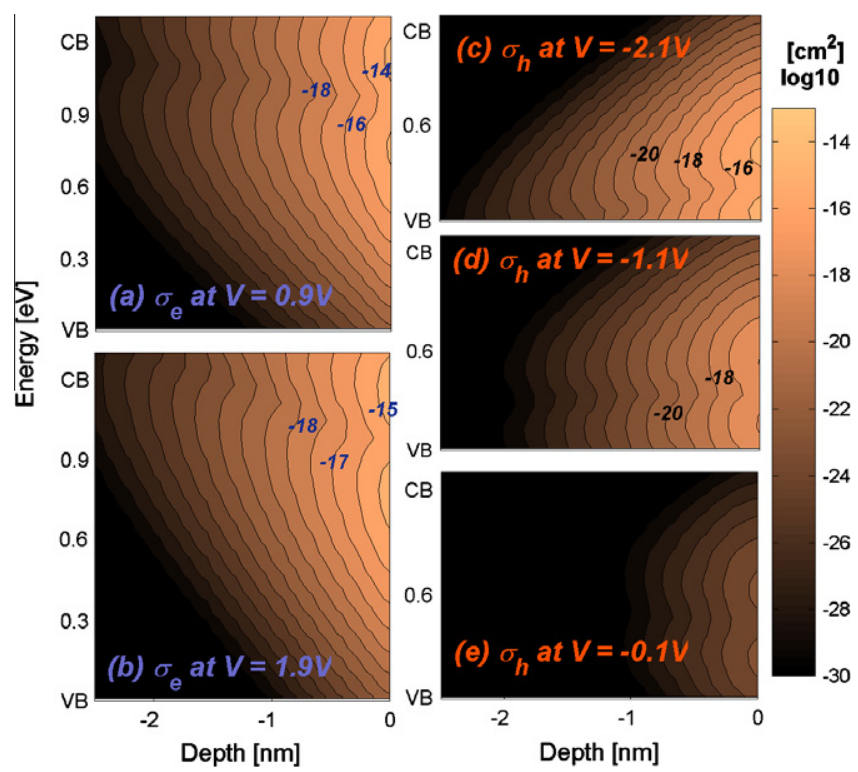

Fig. 4. Capture trap cross-sections of $e^{-}$in (a-b) and $h^{+}$in (c-e) for different bias conditions. The oxide/substrate interface is placed at position $x=0$ with the substrate conduction and valence band edges at $0 \mathrm{eV}$ and $1.2 \mathrm{eV}$. The strong decrease of $\sigma$ with respect to trap energy and position should be taken into account in compact and empirical models to accurately reproduce the defect response and the dynamics of trap filling.
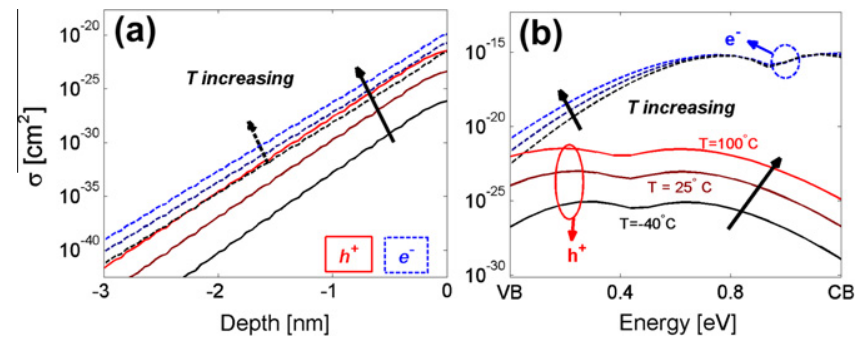

Fig. 5. (a) Trap capture cross-section of $h^{+}$(solid lines) and $e^{-}$(dashed lines) vs. trap depth in proximity of the $\mathrm{Si}$ valence band edge for $V=0.9 \mathrm{~V}$. In the considered multiphonon model, this quantity strongly depends on the device temperature. (b) Energy and temperature dependence of the capture cross-sections at the $\mathrm{Si} / \mathrm{SiO}_{2}$ interface $(x=0)$. The temperature dependence of the $e^{-}$cross-section appears smaller. The temperature ranges from $-40^{\circ} \mathrm{C}$ to $100^{\circ} \mathrm{C}$.

limit at which traps can be characterized with a given small-signal frequency. In depletion, given the large variation of $\mathrm{C} / \mathrm{E}$ rates of several order of magnitudes, only traps located in the vicinity of the Si conduction/valence bands exchange carriers and respond to the AC small-signal. The accessible regions in energy and position can be more quantitatively identified in the cuts in energy and depth shown in Fig. 7a-b. A similar analysis has been performed in depletion $(V=-0.1 \mathrm{~V})$ and for different device temperatures (from $-40^{\circ} \mathrm{C}$ to $100^{\circ} \mathrm{C}$ ). In Fig. $7 \mathrm{c}$ the dependence on oxide depth is shown in proximity of the midgap for $h^{+}$. In Fig. $7 d$, the cut-off frequency exponential variation in trap energy at the $\mathrm{Si} /$ $\mathrm{SiO}_{2}$ interface further illustrates the reduced response of midgap defects in depletion regime. The exponential dependence in temperature is given by the multiphonon assisted C/E rates.

\subsection{Impedance analysis}

Multi-fingered NMOS transistors, having $L=4.8 \mu \mathrm{m}, W=10 \times$ $4 \mu \mathrm{m}$ sizes, and $50 \AA$ oxide thickness, have been integrated in a nanoscale technology and used as reference devices for model
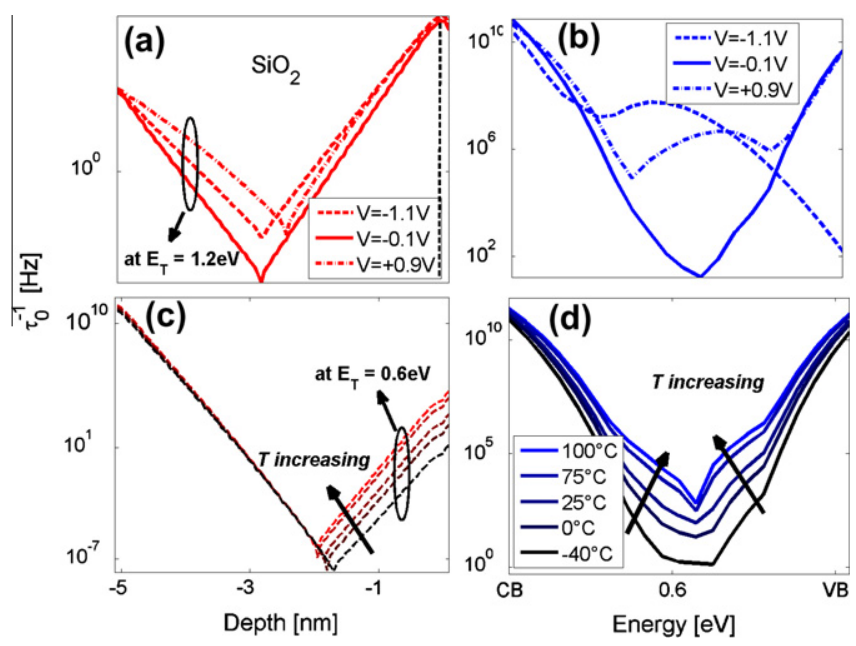

Fig. 7. Depth (a) and energy (b) dependencies of the trap response frequency $\tau_{0}^{-1}$ The cuts correspond to the lines indicated in Fig. 6 for the three considered bias voltages. In (a), $\tau_{0}^{-1}$ is taken at the conduction band level, while in (b) the results are shown at the $\mathrm{Si} / \mathrm{SiO}_{2}$ interface. In depletion (solid line), the exponential decrease of the trap response prevents the characterization of midgap traps, which respond with very low frequencies. (c) Trap cut-off frequency vs. trap depth in the oxide layer for different temperatures from $-40{ }^{\circ} \mathrm{C}$ to $100{ }^{\circ} \mathrm{C}$ and at gate voltage $V=0.9 \mathrm{~V}$. Results in proximity of the midgap are shown. In (d), $\tau_{0}^{-1}$ is shown as a function of defect energy and temperature. The response traps deeper in the oxide layer and in energy exponentially decreases. The frequency response also exponentially increases with temperature. While the temperature dependence does not depend on the position of the trap in the oxide layer, the increase with temperature is more pronounced for defects placed in the midgap.
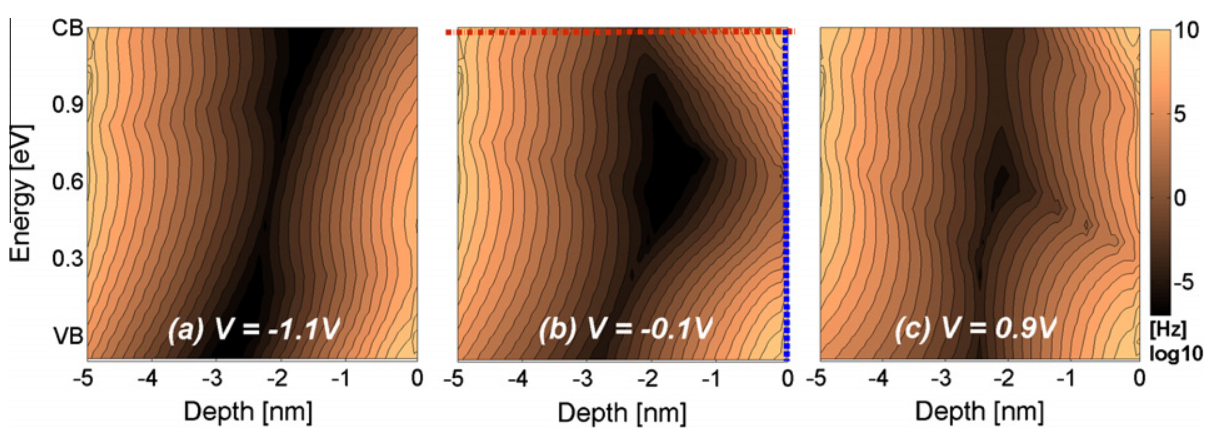

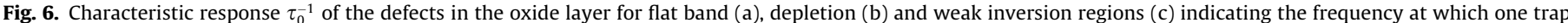

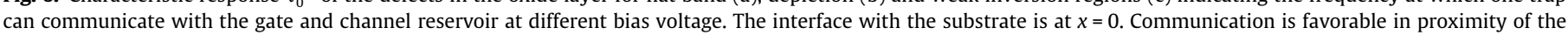

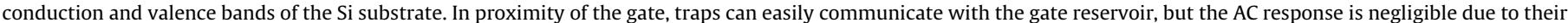
distance from the Si surface. Cuts in energy and depth are shown in Fig. 7. 
validation. AC characteristics (conductance and capacitance) have been measured in a frequency range from $1 \mathrm{kHz}$ to $1 \mathrm{MHz}$ using a HP4284A LCR-meter. The transistors have been subject to electrical stress by applying a positive constant gate voltage of $5.5 \mathrm{~V}$ and keeping the other terminals grounded for $1000 \mathrm{~s}$. After the stress sequence, $\mathrm{CV}$ and $\mathrm{GV}$ characteristics have been measured again to monitor the effects of traps.

Fig. 8 shows the frequency and bias dependences of the measured and simulated channel $C_{\mathrm{GC}}$ and bulk $C_{\mathrm{GB}}$ capacitances of degraded devices. The spatial/energetic defect concentration is a bivariate Gaussian distribution having spatial mean value placed at $2 \AA$ from the $\mathrm{Si} / \mathrm{SiO}_{2}$ interface, the energy mean placed in correspondence of the Si midgap, spatial variance $\sigma_{x}=2 \AA$ and energetic variance $\sigma_{E}=0.6 \mathrm{eV}$. The total defect concentration is $N_{T}=2.05 \times 10^{12} \mathrm{~cm}^{-2}$. These results are consistent with the considerations and quasi-static measurements [32,34].

Several effects attributed to trapping mechanisms emerge, including stretch-out in inversion and accumulation and the intrinsic trap frequency response in depletion and weak inversion [1]. Indeed by decreasing the frequency, deeper traps in energy and

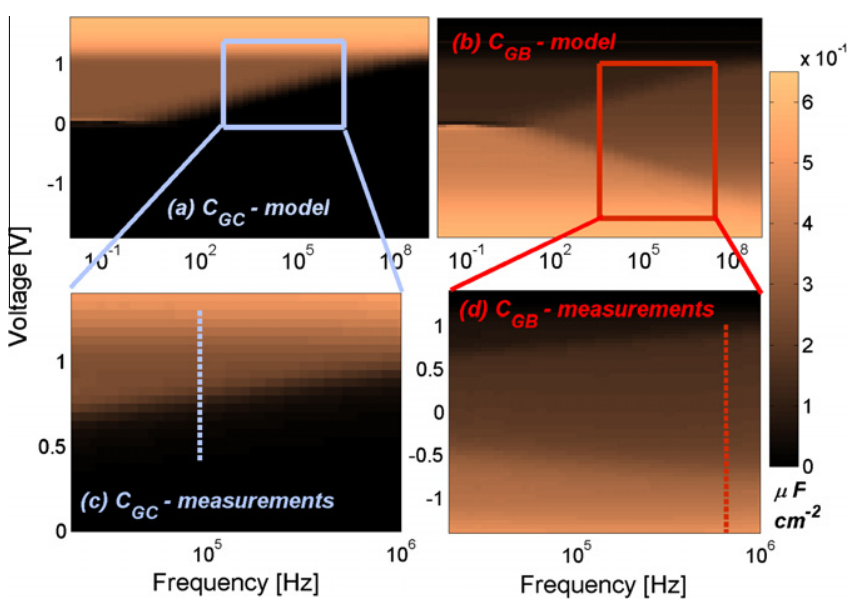

Fig. 8. Channel $(a-c)$ and bulk (b-d) capacitances vs. DC voltage bias and smallsignal frequency. Both $C_{\mathrm{GB}}$ and $C_{\mathrm{GC}}$ indicate the presence of the intrinsic trap response in depletion and weak inversion and two separate bias-dependent cutting frequencies. Simulations (a-b) well reproduce measurement results (c-d) over the entire frequency and bias range. The cut-off frequency on $C_{\mathrm{GB}}$ in weak inversion is the response of the system (bulk charges) to trap filling.

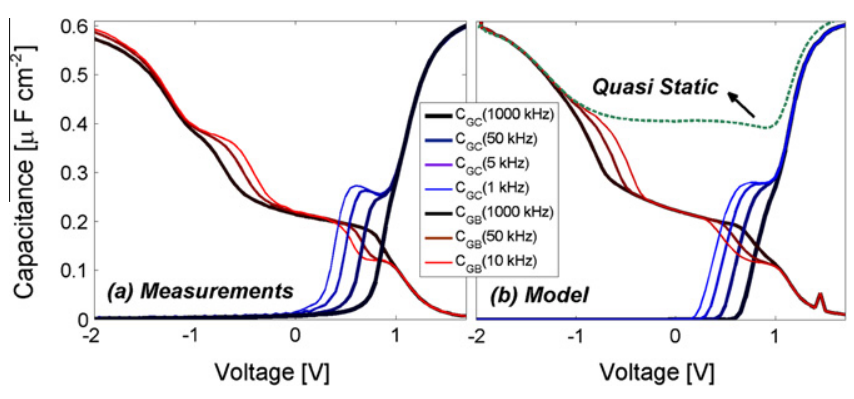

Fig. 9. Channel (in blue) and gate capacitances (in red) vs. applied voltage for several small-signal frequencies. The simulated results in (b) are in fairly good agreement with the measured characteristics. The cuts in frequency from Fig. 8 show the parasitic capacitance due to the trap response added in the region from flat band to weak inversion. This component increases with decreasing frequency as low frequency signals are able to scan the electrical signature of traps having lower $\mathrm{C} / \mathrm{E}$ rates. Indeed, the dashed curve represents the total gate capacitance $C_{\mathrm{GG}}$ in the quasi-static case where all the traps contribute to the $\mathrm{AC}$ response. (For interpretation of the references to color in this figure legend, the reader is referred to the web version of this article.) position are probed, following the iso-lines of $\tau_{0}^{-1}$ as in Fig. 6. Fig. 9 compares the $C_{\mathrm{GB}}$ and $C_{\mathrm{GC}}$ curves for several frequencies and evidences the presence of the parasitic frequency-dependent peaks.

The intrinsic trap capacitance $C_{T}$ of a degraded device and the capacitances of the system due to bulk charges are presented separately in Fig. 10 to illustrate the trap response on the total $C_{\mathrm{GG}}$ capacitance.

The importance of the trap response can be understood considering two extreme cases: at very high frequency, no trap responds to the small-signal voltage, and the trapped charge remains constant to the value of the DC equilibrium condition; at very low frequency, all the traps respond and their intrinsic capacitance response is maximum. The parasitic trap capacitance is added to the system $C_{\mathrm{GG}}$ capacitance, causing an anomalous increase of the total capacitance in depletion. These results are consistent with quasi-static measurements shown in [34], where all the traps are in equilibrium with the substrate.

The dependence of the frequency response of the defects on the critical field $F_{C}$ has been investigated on the trap capacitances and indicates that for values higher than $2 \mathrm{MV} / \mathrm{cm}$ the characteristics are minimally affected, due to the low-field condition in the oxide layer (Fig. 11).

Similar frequency-dependent behavior can be observed on the channel conductance $G_{\mathrm{GB}}$ and bulk conductance $G_{\mathrm{GC}}$, with the
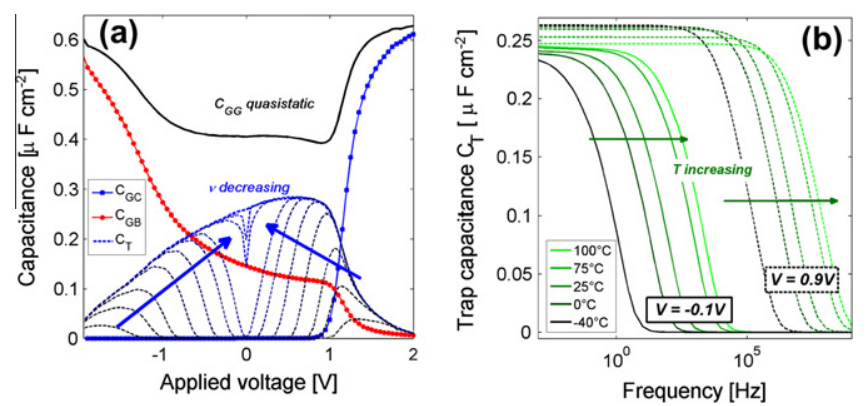

Fig. 10. (a) Simulation results showing the intrinsic parasitic trap capacitance for different bias conditions and frequencies. The parasitic trap contribution $C_{T}$ strongly depends on the frequency of the applied AC signal (dashed lines). At low frequency, all the traps are able to follow the AC component and the response saturates. The parasitic trap component is added to the capacitances of the MOS system (symbols). In quasi-static conditions $(v \rightarrow 0)$ the solid black line of $C_{\mathrm{GG}}$ is obtained. (b) Simulated trap capacitance as a function of small-signal frequency and temperature. Calculations for two voltage conditions are shown. The extracted cut-off frequency corresponds to the $\tau_{0}^{-1}$ frequencies in Fig. 6, determined for the distribution of defects. The exponential variation of three orders of magnitude with temperature varying from $-40{ }^{\circ} \mathrm{C}$ to $100^{\circ} \mathrm{C}$ correlates with the variations of $\tau_{0}^{-1}$ shown in Fig. 7d.

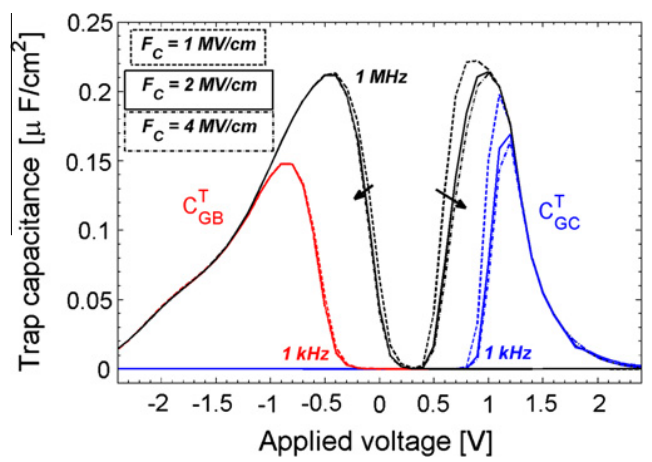

Fig. 11. Trap capacitance vs. applied bias and frequency for different values of the critical field $F_{C}$ and for a low ( $1 \mathrm{kHz}$ ) and high frequency ( $1 \mathrm{MHz}$ ). In the considered device and for the evaluated bias range, values higher than $2 \mathrm{MV} / \mathrm{cm}$ lead to a minimal field dependence. 


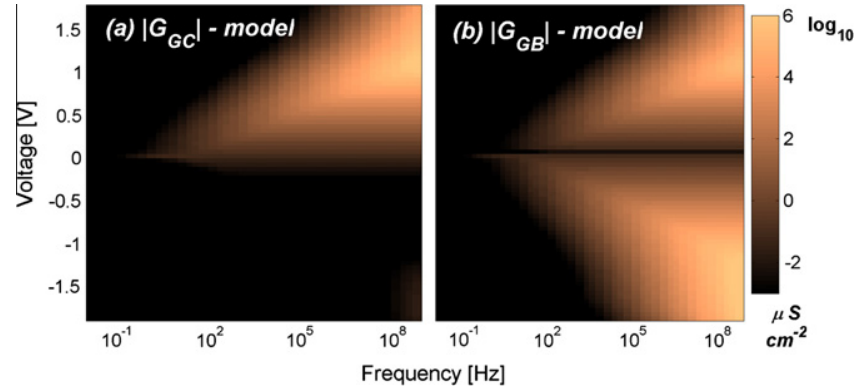

Fig. 12. Simulated channel (a) and bulk (b) conductances vs. DC voltage bias and small-signal frequency. On $\left|G_{\mathrm{GC}}\right|$, the peak present at high frequencies in weak inversion represents the traps $A C$ response and is in close relation with the $C_{\mathrm{GC}}$ capacitance peak in weak inversion. A similar effect is present on $\left|G_{\mathrm{GB}}\right|$ in accumulation. On the other hand, a negative peak is present in weak inversion representing the response of the electrostatics to the trapped charge response.

presence of parasitic peaks at high frequencies representing the response of traps at the interface (Fig. 12).

\section{Conclusion}

A physical based determination of the interface states cross-sections for both $e^{-}$and $h^{+}$has been performed using a multiphononassisted Poisson-Shroedinger approach. Assessing the limitations of neglecting their dependence in energy, position and temperature is important for assessing the approximations introduced in compact reliability models, where an extensive use of constant cross-sections can lead to an inaccurate estimation of the trap concentration distribution. The importance and validity of such a calculation has been highlighted showing its direct application to AC analysis.

\section{References}

[1] Fleetwood D, Winokur P, Reber R, Meisenheimer T, Schwank J, Shaneyfelt M, et al. and border traps on metal-oxide-semiconductor devices. J Appl Phys 1993;73(10):5058-74.

[2] Ielmini D, Manigrasso M, Gattel F, Valentini M. A new NBTI model based on hole trapping and structural relaxation in MOS dielectrics. IEEE Trans Electron Dev 2009;56(9):1943-52.

[3] Palma A, Godoy A, Jimenez-Tejada J, Carceller J, Lopez-Villanueva J. Quantum two-dimensional calculation of time constants of random telegraph signals in metal-oxide-semiconductor structures. Phys Rev B 1997;56(15):9565-74.

[4] Grasser T, Kaczer B, Goes W, Aichinger T, Hehenberger P, Nelhiebel M. Understanding negative bias temperature instability in the context of hole trapping (Invited Paper). Microelectron Eng 2009;86(7-9):1876-82.

[5] Ielmini D, Spinelli A, Rigamonti M, Lacaita A. Modeling of SILC based on electron and hole tunneling. I. Transient effects. IEEE Trans Electron Dev 2002;47(6):1258-65.

[6] Brower K. Dissociation kinetics of hydrogen-passivated (111) $\mathrm{Si}_{-} \mathrm{SiO}_{2}$ interface defects. Phys Rev B 1990;42(6):3444-53.

[7] Nishi Y. Study of silicon-silicon dioxide structure by electron spin resonance. Jpn J Appl Phys 1971;10:52.

[8] Poindexter E, Gerardi G, Rueckel M, Caplan P, Johnson N, Biegelsen D. Electronic traps and $\mathrm{Pb}$ centers at the $\mathrm{Si} / \mathrm{SiO}_{2}$ interface: Band-gap energy distribution. J Appl Phys 2009;56(10):2844-9.
[9] Fleetwood D, Pantelides S, Schrimpf R. Defects in microelectronic materials and devices. CRC; 2008.

[10] Cartier E, Stathis J, Buchanan D. Passivation and depassivation of silicon dangling bonds at the $\mathrm{Si} / \mathrm{SiO}_{2}$ interface by atomic hydrogen. Appl Phys Lett 2009;63(11):1510-2.

[11] Grasser T, Reisinger H, Goes W, Aichinger T, Hehenberger P, Wagner P, et al. Switching oxide traps as the missing link between negative bias temperature instability and random telegraph noise. In: International electron devices meeting. IEEE; 2010. p. 1-4.

[12] Grasser T, Kaczer B. Evidence that two tightly coupled mechanisms are responsible for negative bias temperature instability in oxynitride MOSFETs. IEEE Trans Electron Dev 2009;56(5):1056-62.

[13] Ridley B. Quantum processes in semiconductors. USA: Oxford University Press; 1999.

[14] Ridley B. The photoionisation cross section of deep-level impurities in semiconductors. J Phys C: Solid State Phys 1980;13:2015.

[15] Jiménez-Molinos F, Palma A, Gamiz F, Banqueri J, Lopez-Villanueva J. Physical model for trap-assisted inelastic tunneling in metal-oxide-semiconductor structures. J Appl Phys 2001;90(7):3396-404.

[16] Garetto D, Rideau D, Dornel E, Clark WF, Tavernier C, Leblebici Y, et al. Modeling study of capacitance and gate current in strained highk-metal gate technology. In: Proceedings of 13th international nanotech conference 2010; 2010.

[17] Garetto D, Mamy-Randriamihaja Y, Rideau D, Dornel E, Clark W, Schmid A, et al. Small signal analysis of electrically-stressed oxides with PoissonSchroedinger based multiphonon capture model. In: Proceedings of IEEE international workshop of computational electronics. IEEE; 2010.

[18] Zheng J, Tan H, Ng S. Theory of non-radiative capture of carriers by multiphonon processes for deep centres in semiconductors. J Phys: Condens Matter 1994;6:1695.

[19] Goguenheim D, Lannoo M. Theoretical and experimental aspects of the thermal dependence of electron capture coefficients. J Appl Phys 1990;68(3):1059-69.

[20] Gutsche E. Non-condon approximations and the static approach in the theory of non-radiative multiphonon transitions. Physica Status Solidi (b) 1982;109(2):583-97.

[21] Schenk A. A model for the field and temperature dependence of shockley-readhall lifetimes in silicon. Solid-state Electron 1992;35(11):1585-96.

[22] Hohr T, Schenk A, Fichtner W. Revised shockley-read-hall lifetimes for quantum transport modeling. J Appl Phys 2004;95(9):4875-82.

[23] Huard V. Two independent components modeling for Negative Bias Temperature Instability. In: IRPS symphosium. IEEE; 2010. p. 33-42.

[24] Grasser T, Reisinger H, Wagner P, Schanovsky F, Goes W, Kaczer B. The time dependent defect spectroscopy (TDDS) for the characterization of the bias temperature instability. In: Reliability physics symposium (IRPS), 2010 IEEE international. IEEE; 2010. p. 16-25.

[25] Nguyen T, Mahieu G, Berthe M, Grandidier B, Delerue C, Stiévenard D, et al Coulomb energy determination of a single $\mathrm{Si}$ dangling bond. PRL 2010;105(22):226,404.

[26] Bastard G. Wave mechanics applied to semiconductor heterostructures. Les Ulis, Les Editions de Physique; 1988.

[27] Burt M. The justification for applying the effective-mass approximation to microstructures. J Phys: Condens Matter 1992;4:6651.

[28] Markov S. Gate leakage variability in nano-cmos transistors. Ph.D. thesis University of Glasgow; 2009.

[29] Carrier P, Lewis L, Dharma-Wardana M. Optical properties of structurally relaxed $\mathrm{Si} / \mathrm{SiO}_{2}$ superlattices: The role of bonding at interfaces. Phys Rev B 2002;65(16):165,339.

[30] Grunthaner F, Grunthaner P, Vasquez R, Lewis B, Maserjian J, Madhukar A. High-resolution X-ray photoelectron spectroscopy as a probe of local atomic structure: Application to amorphous $\mathrm{SiO}_{2}$ and the $\mathrm{Si}-\mathrm{SiO}_{2}$ interface. Phys Rev Lett 1979;43(22):1683-6.

[31] Demkov A, Sankey O. Growth study and theoretical investigation of the ultrathin oxide $\mathrm{SiO}_{2}-\mathrm{Si}$ heterojunction. Phys Rev Lett 1999;83(10):2038-41.

[32] Lenahan PM, Dressendorfer PV. Hole traps and trivalent silicon centers in metal/oxide/silicon devices. J Appl Phys 1984;55(10):3495-9.

[33] Heiman F, Warfield G. The effects of oxide traps on the MOS capacitance. IEEE Trans Electron Dev 1985;12(4):167-78.

[34] Fischetti M. Generation of positive charge in silicon dioxide during avalanche and tunnel electron injection. J Appl Phys 1984;57(8):2860-79. 